\title{
Structure Theorems for Systems of Segments
}

\author{
János Pach ${ }^{1}$ and József Solymosi ${ }^{2}$ \\ 1 Rényi Institute of the Hungarian Academy of Sciences \\ and New York University, Courant Institute \\ pach@renyi.hu \\ ${ }^{2}$ Institute for Theoretical Computer Science \\ ETH Zürich \\ solymosi@inf.ethz.ch
}

\begin{abstract}
We study intersection properties of systems of segments in the plane. In particular, we show that there exists a constant $c>0$ such that every system $\mathcal{S}$ of $n$ straight-line segments in the plane has two at least $c n$-element subsystems $\mathcal{S}_{1}, \mathcal{S}_{2} \subset \mathcal{S}$ such that either every segment in $\mathcal{S}_{1}$ intersects all elements of $\mathcal{S}_{2}$, or no segment in $\mathcal{S}_{1}$ intersects any element of $\mathcal{S}_{2}$. We also propose a fast approximate solution for reporting most intersections among $n$ segments in the plane.
\end{abstract}

\section{Introduction}

The problem of detecting and reporting intersections among straight-line segments in the plane is one of the oldest and most extensively studied topics in computational geometry. It is a basic ingredient of many hidden surface removal algorithms, and has numerous other applications in computer graphics, motion planning, geographic information systems, etc. The first efficient techniques were developed by Shamos and Hoey [SH76] and Bentley and Ottmann [BO79] more than twenty years ago. The running times of the best known algorithms, due to Balaban [B95] and Chazelle and Edelsbrunner [CE92], are $O(n \log n+I)$, where $n$ and $I$ denote the number of segments and the number of intersections, resp. (See also [PS91].)

In the present paper, we discuss some structural properties of intersection graphs of segments, i.e., graphs that can be obtained by assigning a vertex to every element of a system of segments $\mathcal{S}$ in the plane, and connecting two of them by an edge if and only if their intersection is non-empty. Throughout this paper, we assume that the elements of $\mathcal{S}$ are in general position, i.e., no two segments are parallel and no three of their endpoints are collinear. In particular, if two elements of $\mathcal{S}$ intersect, then they determine a proper crossing.

We prove the following Ramsey-type result.

\footnotetext{
${ }^{1}$ Supported by NSF grant CR-97-32101, PSC-CUNY Research Award 61392-0030, and OTKA-T-020914.

${ }^{2}$ Supported by the joint Berlin/Zürich graduate program Combinatorics, Geometry, Computation, financed by German Science Foundation (DFG) and ETH Zürich. On leave from MTA SZTAKI
} 
Theorem 1. There exists a constant $C>0$ such that every system $\mathcal{S}$ of $n$ segments in the plane has two disjoint subsystems $\mathcal{S}_{1}, \mathcal{S}_{2} \subset \mathcal{S}$ such that $\left|\mathcal{S}_{1}\right|,\left|\mathcal{S}_{2}\right| \geq C n$ and

(i) either every segment in $\mathcal{S}_{1}$ crosses all segments in $\mathcal{S}_{2}$,

(ii) or no segment in $\mathcal{S}_{1}$ crosses any segment in $\mathcal{S}_{2}$.

In the sequel, $A$ stands for an absolute constant smaller than $10^{6}$. Theorem 1 is a direct corollary of the following two complementary results.

Theorem 2. Any system $\mathcal{S}$ of $n$ segments in the plane with at least $\mathrm{cn}^{2}$ crossings $(c>0)$ has two disjoint subsystems, $\mathcal{S}_{1}, \mathcal{S}_{2} \subset \mathcal{S}$, such that $\left|\mathcal{S}_{1}\right|,\left|\mathcal{S}_{2}\right| \geq \frac{(2 c)^{A}}{660} n$ and every segment in $\mathcal{S}_{1}$ crosses all segments in $\mathcal{S}_{2}$.

Theorem 3. Any system $\mathcal{S}$ of $n$ segments in the plane with at least $\mathrm{cn}^{2}$ noncrossing pairs $(c>0)$ has two disjoint subsystems, $\mathcal{S}_{1}, \mathcal{S}_{2} \subset \mathcal{S}$, such that $\left|\mathcal{S}_{1}\right|,\left|\mathcal{S}_{2}\right|$ $\geq \frac{(c / 5)^{A}}{330} n$ and no segment in $\mathcal{S}_{1}$ crosses any segment in $\mathcal{S}_{2}$.

The above results, combined with Szemerédi's Regularity Lemma [S78], can be used to establish a fairly strong structure theorem for intersection graphs of segments. We say that two sets have almost the same number of elements if their sizes differ by at most a factor of 2 .

Theorem 4. For any $\varepsilon>0$, there exists an integer $K=K(\varepsilon)$ with the property that any system $\mathcal{S}$ of segments in the plane can be partitioned into $K+1$ subfamilies, $\mathcal{S}_{0}, \mathcal{S}_{1}, \ldots, \mathcal{S}_{K}$ such that $\left|\mathcal{S}_{0}\right|<\varepsilon|\mathcal{S}|$, all other subfamilies have almost the same size, and for all but at most $\varepsilon K^{2}$ pairs $1 \leq i, j \leq K$,

(i) either every segment in $\mathcal{S}_{i}$ crosses all segments in $\mathcal{S}_{j}$,

(ii) or no segment in $\mathcal{S}_{i}$ crosses any segment in $\mathcal{S}_{j}$.

Fix an element $s_{i}$ in each $\mathcal{S}_{i}$. For any $s \in \mathcal{S}$, let $f(s):=s_{i}$ if and only if $s$ belongs $\mathcal{S}_{i}(0 \leq i \leq K)$. We can think of $f(s)$ as the segment representing $s$. According to Theorem 4 , with a very small error, two randomly selected elements $s, t \in \mathcal{S}$ cross each other if and only if $f(s) \cap f(t)$ is non-empty.

A geometric graph is a graph whose vertices are points in general position in the plane (i.e., no three points are on a line) and whose edges are straight-line segments connecting these points. Our last two results are easy corollaries to Theorems 2 and 3 , respectively.

Theorem 5. Any geometric graph $G$ with $n$ vertices and at least $c n^{2}$ edges $(c>0)$ has two disjoint sets of edges $E_{1}, E_{2} \subset E(G)$ such that $\left|E_{1}\right|,\left|E_{2}\right| \geq$ $(c / 32)^{A+3}\left(\begin{array}{l}n \\ 2\end{array}\right)$ and every edge in $E_{1}$ crosses all edges in $E_{2}$.

Theorem 6. Any geometric graph $G$ with $n$ vertices and at least $\mathrm{cn}^{2}$ edges $(c>0)$ has two disjoint sets of edges $E_{1}, E_{2} \subset E(G)$ such that $\left|E_{1}\right|,\left|E_{2}\right| \geq$ $(c / 34)^{A+3}\left(\begin{array}{c}n \\ 2\end{array}\right)$ and no edge in $E_{1}$ crosses any edge in $E_{2}$.

The rest of the paper is organized as follows. In Section 2, we establish Theorems 2 and 3. Theorem 4 is proved in Section 3. The last section contains the proofs of Theorems 5 and 6 , as well as some concluding remarks. 


\section{Proofs of Theorems 2 and 3}

Three sets of points in the plane are said to be separable if each of them can be separated from the other two by a straight line. Given three separable sets, there is no straight line which intersects the convex hull of all of them.

Lemma 1. Every set of $n$ points in general position in the plane has three separable subsets of size $\lfloor n / 6\rfloor$.

Proof. Assume without loss of generality that $n$ is divisible by 6 , and let $P$ be an $n$-element point set. Choose two lines that divide the plane into 4 regions, containing $n, 2 n, n$, and $2 n$ points of $P$ in their interiors, in this cyclic order. Let $P_{1}, P_{2}, P_{3}$, and $P_{4}$ denote the corresponding subsets of $P$. By the ham-sandwich theorem, there is a line $\ell$ which simultaneously cuts $P_{2}$ and $P_{4}$ into two halves of equal size (see Fig. 1). Then $\ell$ avoids either the convex hull of $P_{1}$ or that of $P_{3}$. Assume, by symmetry, that $P_{1}$ is 'above' $\ell$. Then $P_{1}$ and the parts of $P_{2}$ and $P_{4}$ 'below' $\ell$ are three separable sets.

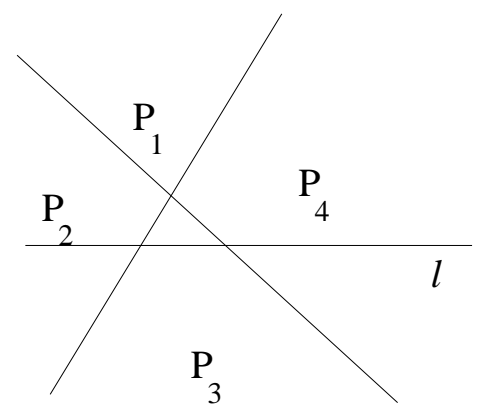

Fig. 1.

Lemma 2. Let $\mathcal{S}$ and $\mathcal{T}$ be two systems of segments in general position in the plane. Then there are two subsystems $\mathcal{S}^{*} \subseteq \mathcal{S}, \mathcal{T}^{*} \subseteq \mathcal{T}$ such that $\left|\mathcal{S}^{*}\right| \geq$ $\lfloor|\mathcal{S}| / 330\rfloor,\left|\mathcal{T}^{*}\right| \geq\lfloor|\mathcal{T}| / 330\rfloor$, and

(i) either every segment in $\mathcal{S}^{*}$ crosses all segments in $\mathcal{T}^{*}$,

(ii) or no segment in $\mathcal{S}^{*}$ crosses any segment in $\mathcal{T}^{*}$.

Proof. Let $|\mathcal{S}|=m,|\mathcal{T}|=n$, and suppose, for simplicity, that both $m$ and $n$ are multiples of 330. Let $P$ be the set of endpoints of all segments in $\mathcal{S}$. By Lemma 1, there are three separable $m / 3$-element subsets, $P_{1}, P_{2}, P_{3} \subseteq P$. Color a segment $t \in \mathcal{T}$ with color $i$ if its supporting line does not intersect the convex hull of $P_{i}(i=1,2,3)$. Let $\mathcal{T}_{i}$ denote the segments of color $i$. At least one third of the 
elements of $\mathcal{T}$ get the same color, so we can assume with no loss of generality that $\left|\mathcal{T}_{1}\right| \geq n / 3$.

If there are at least $m / 330$ segments in $\mathcal{S}$, both of whose endpoints belong to $P_{1}$, then we are done, because these segments are disjoint from all elements of $\mathcal{T}_{1}$.

Hence, we can assume that at least $(1 / 3-2 / 330) m=18 m / 55$ elements of $\mathcal{S}$ have precisely one of their endpoints in $P_{1}$. Let $Q$ denote the set of other endpoints of these segments. Let us choose three separable subsets $Q_{1}, Q_{2}, Q_{3} \subseteq$ $Q$, each of size at least $|Q| / 6=3 \mathrm{~m} / 55$. Just as before, color a segment $t \in \mathcal{T}_{1}$ with color $i$ if its supporting line does not intersect the convex hull of $Q_{i}(i=1,2,3)$. Again, at least $\left|\mathcal{T}_{1}\right| / 3 \geq n / 9$ elements of $\mathcal{T}_{1}$ get the same color, say color 1 ; they form a subsystem $\mathcal{T}_{11} \subseteq \mathcal{T}_{1}$.

Let $\mathcal{S}_{11}$ denote set of all elements of $\mathcal{S}$ with one endpoint in $P_{1}$ and the other in $Q_{1}$. Clearly, we have $\left|\mathcal{S}_{11}\right|=\left|Q_{1}\right| \geq 3 \mathrm{~m} / 55$.

Let us repeat now the whole procedure with $\mathcal{T}_{11}$ in the place of $\mathcal{S}$ and $\mathcal{S}_{11}$ in the place of $\mathcal{T}$. We obtain two subsets, $\mathcal{T}^{\prime} \subseteq \mathcal{T}_{11}$ and $\mathcal{S}^{\prime} \subseteq \mathcal{S}_{11}$, satisfying

$$
\left|\mathcal{T}^{\prime}\right| \geq \frac{3\left|\mathcal{T}_{11}\right|}{55} \geq \frac{n}{165}, \quad\left|\mathcal{S}^{\prime}\right| \geq \frac{\left|\mathcal{S}_{11}\right|}{9} \geq \frac{m}{165} .
$$

We can assume that at least half of the supporting lines of the elements of $\mathcal{T}^{\prime}$ cross the convex hull of $\mathcal{S}^{\prime}$, for otherwise we would obtain two non-crossing systems of at least $\left|\mathcal{T}^{\prime}\right| / 2$ and $\left|\mathcal{S}^{\prime}\right|$ segments. The set of of all elements of $\mathcal{T}^{\prime}$, whose supporting lines cross the convex hull of $\mathcal{S}^{\prime}$ is denoted by $\mathcal{T}^{*}$. Similarly, we can assume that the supporting lines of at least half of the elements of $\mathcal{S}^{\prime}$ cross the convex hull of $\mathcal{T}^{*}$; otherwise, we could find two non-crossing systems of at least $\left|\mathcal{T}^{*}\right|$ and $\left|\mathcal{S}^{\prime}\right| / 2$ segments. Let $\mathcal{S}^{*}$ denote the set of all elements of $\mathcal{S}^{\prime}$, whose supporting lines cross the convex hull of $\mathcal{T}^{*}$. It follows from the definitions that every element of $\mathcal{S}^{*}$ crosses all elements of $\mathcal{T}^{*}$ and that

$$
\left|\mathcal{S}^{*}\right| \geq \frac{|\mathcal{S}|^{\prime}}{2} \geq \frac{m}{330}, \quad\left|\mathcal{T}^{*}\right| \geq \frac{|\mathcal{T}|^{\prime}}{2} \geq \frac{n}{330} .
$$

Given any system of segments, $\mathcal{S}$ and $\mathcal{T}$, in general position in the plane, define their crossing density, $\delta(\mathcal{S}, \mathcal{T})$, as the number of crossing pairs $(s, t), s \in$ $\mathcal{S}, t \in \mathcal{T}$ divided by $|\mathcal{S}| \cdot|\mathcal{T}|$. Clearly, we have $0 \leq \delta(\mathcal{S}, \mathcal{T}) \leq 1$.

Theorems 2 and 3 readily follow from the next result.

Theorem 7. There exists a constant $A<10^{6}$ satisfying the following condition. Let $\mathcal{S}$ and $\mathcal{T}$ be any sets of segments in general position in the plane, and suppose that their crossing density is at least $c>0$. Then there are two disjoint subsystems $\mathcal{S}^{\prime} \subseteq \mathcal{S}, \mathcal{T}^{\prime} \subseteq \mathcal{T}$ such that

$$
\left|\mathcal{S}^{\prime}\right| \geq \frac{c^{A}}{330}|\mathcal{S}|, \quad\left|\mathcal{T}^{\prime}\right| \geq \frac{c^{A}}{330}|\mathcal{T}|
$$

and every segment in $\mathcal{S}^{\prime}$ crosses all segments in $\mathcal{T}^{\prime}$. 
Proof. Let $|\mathcal{S}|=m,|\mathcal{T}|=n$, and suppose first that both $m$ and $n$ are powers of 330. According to our assumption, $\delta(\mathcal{S}, \mathcal{T}) \geq c$.

Applying Lemma 2, we obtain two subsystems, $\mathcal{S}^{*} \subset \mathcal{S}, \mathcal{T}^{*} \subset \mathcal{T}$, such that $\left|\mathcal{S}^{*}\right|=m / 330,\left|\mathcal{T}^{*}\right|=n / 330$, and $\delta\left(\mathcal{S}^{*}, \mathcal{T}^{*}\right)$ is either 1 or 0 . In the first case we are done, so assume $\delta\left(\mathcal{S}^{*}, \mathcal{T}^{*}\right)=0$. Then we have

$c \leq \delta(\mathcal{S}, \mathcal{T})=\frac{329}{330^{2}} \delta\left(\mathcal{S}, \mathcal{T}-\mathcal{T}^{*}\right)+\frac{329}{330^{2}} \delta\left(\mathcal{S}-\mathcal{S}^{*}, \mathcal{T}\right)+\frac{329^{2}}{330^{2}} \delta\left(\mathcal{S}-\mathcal{S}^{*}, \mathcal{T}-\mathcal{T}^{*}\right)$.

Therefore, at least one of the crossing densities $\delta\left(\mathcal{S}, \mathcal{T}-\mathcal{T}^{*}\right), \delta\left(\mathcal{S}-\mathcal{S}^{*}, \mathcal{T}\right)$, $\delta\left(\mathcal{S}-\mathcal{S}^{*}, \mathcal{T}-\mathcal{T}^{*}\right)$ exceeds

$$
c_{1}:=c \frac{330^{2}}{330^{2}-1} .
$$

In other words, there exist two subsystems, $\mathcal{S}_{1} \subset \mathcal{S}, \mathcal{T}_{1} \subset \mathcal{T}$, with $\left|\mathcal{S}_{1}\right| \geq m / 330$, $\left|\mathcal{T}_{1}\right| \geq n / 330$ such that $\delta\left(\mathcal{S}_{1}, \mathcal{T}_{1}\right) \geq c_{1}$.

Applying Lemma 2 to $\mathcal{S}_{1}$ and $\mathcal{T}_{1}$, we obtain two subsystems $\mathcal{S}^{* *} \subset \mathcal{S}_{1}, \mathcal{T}^{* *} \subset$ $\mathcal{T}_{1}$, such that $\left|\mathcal{S}^{* *}\right| \geq m / 330^{2},\left|\mathcal{T}^{* *}\right| \geq n / 330^{2}$, and $\delta\left(\mathcal{S}^{* *}, \mathcal{T}^{* *}\right)$ is either 1 or 0 . Again, we can assume that $\delta\left(\mathcal{S}^{* *}, \mathcal{T}^{* *}\right)=0$, otherwise we are done. As before, we can find two subsystems, $\mathcal{S}_{2} \subset \mathcal{S}_{1}, \mathcal{T}_{2} \subset \mathcal{T}_{1}$, with $\left|\mathcal{S}_{2}\right| \geq m / 330^{2},\left|\mathcal{T}_{2}\right| \geq n / 330^{2}$ such that

$$
\delta\left(\mathcal{S}_{2}, \mathcal{T}_{2}\right) \geq c_{2}:=c\left(\frac{330^{2}}{330^{2}-1}\right)^{2} .
$$

Since the crossing density between any two sets is at most 1 , after some

$$
k \leq \frac{\log \frac{1}{c}}{\log \frac{330^{2}}{330^{2}-1}}
$$

steps, this procedure will terminate. That is, when we apply Lemma 2 for the $k$-th time, we obtain two subsystems $\mathcal{S}^{\prime} \subseteq \mathcal{S}, \mathcal{T}^{\prime} \subseteq \mathcal{T}$ such that $\left|\mathcal{S}^{\prime}\right| \geq m / 330^{k}$, $\left|\mathcal{T}^{\prime}\right| \geq n / 330^{k}$, and $\delta\left(\mathcal{S}^{\prime}, \mathcal{T}^{\prime}\right)=1$. Thus, every element of $\mathcal{S}^{\prime}$ crosses all elements of $\mathcal{T}^{\prime}$, and $\left|\mathcal{S}^{\prime}\right| \geq c^{A} m,\left|\mathcal{T}^{\prime}\right| \geq c^{A} n$, where

$$
A \leq \frac{\log 330}{\log \frac{330^{2}}{330^{2}-1}}<10^{6} .
$$

This completes the proof of Theorem 7 in the case when $m$ and $n$ are powers of 330. Otherwise, using an easy averaging argument, we can find $\mathcal{S}_{0} \subseteq \mathcal{S}, \mathcal{T}_{0} \subseteq \mathcal{T}$, whose sizes are powers of $330,\left|\mathcal{S}_{0}\right| \geq m / 330,\left|\mathcal{T}_{0}\right| \geq n / 330$, and $\delta\left(\mathcal{S}_{0}, \mathcal{T}_{0}\right) \geq c$. Applying the above argument to $\mathcal{S}_{0}$ and $\mathcal{T}_{0}$, the result follows.

Proof of Theorem 2. Assume, for simplicity, that $n$ is even. Given a system of $n$ segments in general position in the plane, which determine at least $\mathrm{cn}^{2}$ crossings, one can partition it into two equal parts so that the crossing density between them is at least $2 c$ (see e.g. [PA95]). Applying Theorem 7 to these parts, the result follows. 
Theorem 3 can be established analogously, by repeated application of Lemma 2. However, here we deduce it from Theorems 2 and 3.

Proof of Theorem 3. Let $\mathcal{S}$ be a set of $n$ segments in general position in the plane with at least $c n^{2}$ non-crossing pairs. For any $s \in \mathcal{S}$, let $\ell(s)$ denote the supporting line of $s$. The set $\ell(s) \backslash s$ consists of two half-lines; denote them by $h_{1}(s)$ and $h_{2}(s)$. Let $\mathcal{H}_{1}:=\left\{h_{1}(s): s \in \mathcal{S}\right\}, \mathcal{H}_{2}:=\left\{h_{2}(s): s \in \mathcal{S}\right\}, \mathcal{T}:=\mathcal{S} \cup \mathcal{H}_{1} \cup \mathcal{H}_{2}$. Further, for any $h \in \mathcal{H}_{1} \cup \mathcal{H}_{2}$, let $s(h)$ be the unique segment $s \in \mathcal{S}$, for which $h_{1}(s)$ or $h_{2}(s)$ is equal to $s$.

Note that if two segments $s, t \in \mathcal{S}$ do not cross each other, then the crossing between their supporting lines, $\ell(s)$ and $\ell(t)$, gives rise to a crossing between a pair of elements of $\mathcal{T}$, involving at least one half-line. Therefore, the number of crossing pairs in $\mathcal{T}$ involving at least one half-line is at least $c n^{2}$. There are three possibilities:

1. for some $i=1,2$, the number of crossing pairs in $\mathcal{H}_{i}$ is at least $c n^{2} / 5$;

2. the number of crossing pairs between $\mathcal{H}_{1}$ and $\mathcal{H}_{2}$ is at least $c n^{2} / 5$;

3. for some $i=1,2$, the number of crossing pairs between $\mathcal{H}_{i}$ and $\mathcal{S}$ is at least $c n^{2} / 5$.

In Case 1, applying Theorem 2 to $\mathcal{H}_{i}$, we obtain two subsystems, $\mathcal{H}_{i 1}, \mathcal{H}_{i 2} \subset$ $\mathcal{H}$, whose sizes are at least $\frac{(2 c / 5)^{A}}{660} n>\frac{(c / 5)^{A}}{330}$, and every half-line in $\mathcal{H}_{i 1}$ crosses all half-lines in $\mathcal{H}_{i 2}$. Then $\mathcal{S}_{1}:=\left\{s(h): h \in \mathcal{H}_{i 1}\right\}$ and $\mathcal{S}_{2}:=\left\{s(h): h \in \mathcal{H}_{i 2}\right\}$ meet the requirements in Theorem 3 .

In Case 2, apply Theorem 7 to obtain $\mathcal{H}_{1}^{\prime} \subseteq \mathcal{H}_{1}, \mathcal{H}_{2}^{\prime} \subseteq \mathcal{H}_{2}$, whose sizes are at least $\frac{(c / 5)^{A}}{330} n$, and every element of $\mathcal{H}_{1}^{\prime}$ crosses all elements of $\mathcal{H}_{2}^{\prime}$. Setting $\mathcal{S}_{1}:=\left\{s(h): h \in \mathcal{H}_{1}^{\prime}\right\}$, and $\mathcal{S}_{2}:=\left\{s(h): h \in \mathcal{H}_{2}^{\prime}\right\}$, the result follows. Case 3 can be treated similarly.

\section{Proof of Theorem 4}

The proof is based on a variant of Szemerédi's Regularity Lemma, which was discovered by Komlós (see [KS96]) and can be established by an elegant argument.

For any graph $G$ and for any disjoint subsets $X, Y \subset V(G)$, let $E(X, Y) \subseteq$ $E(G)$ denote the set of edges of $G$ running between $X$ and $Y$. Clearly, we have $|E(X, Y)| \leq|X||Y|$. For any $\gamma, \delta>0$, we call the pair $(X, Y)(\gamma, \delta)$-superregular if for every $X^{\prime} \subseteq X$ and $Y^{\prime} \subseteq Y$ satisfying

$$
\left|X^{\prime}\right| \geq \gamma|X|, \quad\left|Y^{\prime}\right| \geq \gamma|Y|
$$

we have

$$
\left|E\left(X^{\prime}, Y^{\prime}\right)\right| \geq \delta|X||Y|
$$

Lemma 3. ([KS96]) Let $\gamma>0$ be a sufficiently small constant, and let $\delta>0$. 
Then any graph with $n$ vertices and at least $\delta n^{2}$ edges has a $(\gamma, \delta)$-superregular pair $(X, Y)$ with

$$
|X|=|Y| \geq \delta^{1 / \gamma^{2}} n .
$$

First we establish

Theorem 8. For every $\delta>0$, there exists an integer $k=k(\delta)>0$ with the following property. The intersection graph $G$ of any system of $n$ segments in the plane has $k$ bipartite subgraphs, which altogether cover all but at most $\delta n^{2}$ edges of $G$.

Proof. Set $G_{0}:=G$, and let $\beta$ be a small positive constant to be specified later. Suppose that for some $i \geq 1$ we have already defined $G_{i-1}$. If $G$ does not have a complete bipartite subgraph $H_{i}$, which contains at least $\beta n^{2}$ edges of $G_{i-1}$, then stop. Otherwise, pick such a subgraph $H_{i}$, and let $G_{i}$ denote the graph obtained from $G_{i-1}$ by the deletion of all edges belonging to $H_{i}$. Obviously, this procedure will terminate in

$$
j \leq \frac{|E(G)|}{\beta n^{2}} \leq \frac{1}{2 \beta}
$$

steps, with a graph $G_{j}$.

We claim that $G_{j}$ has fewer than $\delta n^{2}$ edges, provided that $\beta$ is sufficiently small. Suppose that this is not true. Then, according to Lemma $3, G_{j}$ has a $(\gamma, \delta)$-superregular pair $(X, Y)$ with

$$
|X|=|Y| \geq \delta^{1 / \gamma^{2}} n,
$$

where $\gamma:=\frac{\delta^{A}}{330}$. Let $\mathcal{S}_{X}$ and $\mathcal{S}_{Y}$ denote the corresponding families of segments. In view of Theorem 7 , there are two disjoint subsystems $\mathcal{T}_{X} \subseteq \mathcal{S}_{X}$ and $\mathcal{T}_{Y} \subseteq \mathcal{S}_{Y}$ with

$$
\left|\mathcal{T}_{X}\right|=\left|\mathcal{T}_{Y}\right| \geq \frac{\delta^{A}}{330}|X|=\gamma|X|=\gamma|Y|
$$

such that every segment in $\mathcal{T}_{X}$ crosses all elements of $\mathcal{T}_{Y}$. Let $X^{\prime}$ and $Y^{\prime}$ denote the subsets of $X$ and $Y$, corresponding to $\mathcal{T}_{X}$ and $\mathcal{T}_{Y}$, resp. Then $X^{\prime}$ and $Y^{\prime}$ induce a complete bipartite subgraph in $G$. Furthermore, using the fact that $(X, Y)$ is a $(\gamma, \delta)$-superregular pair in $G_{j}$, we obtain that at least

$$
\delta\left|X^{\prime}\right|\left|Y^{\prime}\right| \geq \delta \gamma^{2} \delta^{2 / \gamma^{2}} n^{2}
$$

edges between $X^{\prime}$ and $Y^{\prime}$ belong to $G_{j}$. Therefore, if we choose $\beta$ so small that this last quantity exceeds $\beta n^{2}$, then we could continue our procedure and define the next graph $G_{j+1}$. This contradiction completes the proof.

Obviously, a similar result holds for $\bar{G}$, the complement of a segment intersection graph $G$. 
Theorem 9. For every $\delta>0$, there exists an integer $k=k(\delta)>0$ with the following property. The complement $\bar{G}$ of the intersection graph $G$ of any system of $n$ segments in in the plane has $k$ bipartite subgraphs, which altogether cover all but at most $\delta n^{2}$ edges of $\bar{G}$.

Now we are in a position to prove Theorem 4 . Let $G$ denote the intersection graph of $\mathcal{S}$. Let $\delta$ be a small positive constant which will be specified later. By Theorems 8 and 9 , there is a family

$$
\mathcal{F}=\left\{A_{1}, B_{1}, A_{2}, B_{2}, \ldots, A_{2 k}, B_{2 k}\right\}
$$

of subsets of $V(G)$ such that

1. $A_{i}$ and $B_{i}$ are disjoint $(1 \leq i \leq 2 k)$;

2. $A_{i} \times B_{i}$ is contained either in $E(G)$ or in $E(\bar{G}) \quad(1 \leq i \leq 2 k)$;

3. all but at most $2 \delta n^{2}$ pairs $\{u, v\} \subset V(G)$ are covered by $\cup_{i=1}^{2 k} A_{i} \times B_{i}$.

We say that two vertices of $V(G)$ are of the same type, if every member of $\mathcal{F}$ contains both or neither of them. The number of different types is at most $3^{2 k}$. A given type is negligible, if fewer than

$$
s:=\frac{\varepsilon n}{3^{2 k}}
$$

vertices have it. Letting $V_{0}$ denote the set of all vertices with negligible types, we have $\left|V_{0}\right|<\varepsilon n$.

Divide the elements of $V(G)-V_{0}$ into groups $V_{1}, V_{2}, \ldots, V_{K}$ of almost the same size: for every $1 \leq i \leq K$, let $s \leq\left|V_{i}\right| \leq 2 s$. Clearly, we have

$$
\frac{(1-\varepsilon) n}{2 s} \leq K \leq \frac{n}{s}
$$

A pair $(i, j), 1 \leq i \neq j \leq K$ is called exceptional, if $V_{i} \times V_{j}$ is not contained in $\cup_{i=1}^{2 k} A_{i} \times B_{i}$. For every non-exceptional pair $(i, j), V_{i}$ and $V_{j}$ induce a complete bipartite subgraph either in $G$ or in $\bar{G}$.

Let $m$ denote the number of exceptional pairs. The total number of pairs $\{u, v\} \subset V(G)$ for which $u \in V_{i}, v \in V_{j}$ for some exceptional pair $(i, j)$ is at least $m s^{2}$. On the other hand, by condition 3 above, this number cannot exceed $2 \delta n^{2}$. Thus, we obtain that

$$
\frac{m}{K^{2}} \leq \frac{2 \delta n^{2}}{s^{2} K^{2}} \leq \frac{2 \delta n^{2}}{s^{2}} \frac{4 s^{2}}{(1-\varepsilon)^{2} n^{2}}=\frac{8 \delta}{(1-\varepsilon)^{2}} .
$$

This is smaller than $\varepsilon$, if $\delta$ is sufficiently small, so the partition of $\mathcal{S}$ corresponding to $V_{0} \cup V_{1} \cup \ldots \cup V_{K}$ meets all the requirements of Theorem 4 .

\section{Concluding remarks}

First we show how Theorems 5 and 6 follow from the previous results. 
Proof of Theorem 5. Let $G$ be a geometric graph with $n$ vertices and at least $c n^{2}$ edges. The next result of Ajtai, Chvátal, Newborn, Szemerédi [ACNS82] and, independently, Leighton [L83] (see also [PA95], [PT97]) implies that there are at least $\frac{c}{64} e^{2}$ crossings pairs of edges.

Lemma 4. Let $G$ be a geometric graph with $n$ vertices and $e>4 n$ edges, for some $c>0$. Then $G$ has at least $\frac{e^{3}}{64 n^{2}}$ crossing pairs of edges.

Thus, we can apply Theorem 2 to the system $\mathcal{S}=E(G)$. We obtain two subsets $E_{1}, E_{2} \in E(G)$ such that every edge in $E_{1}$ crosses all edges in $E_{2}$, and $\left|E_{1}\right|=\left|E_{2}\right| \geq \frac{(c / 32)^{A}}{336} c n^{2}>(c / 32)^{A+2}\left(\begin{array}{c}n \\ 2\end{array}\right)$.

Theorem 6 can be proved similarly. The only difference is that, instead of Theorem 2 and Lemma 4, we have to use Theorem 3 and

Lemma 5. ([P91]) Let $G$ be a geometric graph with $n$ vertices and $e \geq 3 n / 2$ edges, for some $c>0$. Then $G$ has at least $\frac{4 e^{3}}{27 n^{2}}$ pairs of edges that do not cross and do not share an endpoint.

The above theorems can also be established using Szemerédi's Regularity Lemma [S78]. However, then the dependence on $c$ of the sizes of the homogeneous subsystems whose existence is guaranteed by our results gets much worse.

According to an old theorem of Kövári, Sós, and Turán [KST54], every graph with $n$ vertices and at least $c n^{2}$ edges has a complete bipartite subgraph with $c^{\prime} \log n$ vertices in its classes, where $c^{\prime}>0$ is a suitable constant depending on $c$. This immediately implies that Theorem 2 holds with the much weaker bound $c^{\prime} \log n$ instead of $c^{\prime} n$.

For some computational aspects of recognizing intersection graphs of segments, see [KM94].

\section{References}

[ACNS82] M. Ajtai, V. Chvátal, M. Newborn, and E. Szemerédi, Crossing-free subgraphs, Annals of Discrete Mathematics 12 (1982), 9-12.

[B95] I. J. Balaban, An optimal algorithm for finding segment intersections, in: Proc. 11th Annual ACM Symposium on Computational Geometry, ACM, 1995, 211219.

[BO79] J. L. Bentley and T. A. Ottmann, Algorithms for reporting and counting geometric intersections, IEEE Trans. Comput. C-28 (1979), 643-647.

[CE92] B. Chazelle and H. Edelsbrunner, An optimal algorithm for intersecting line segments in the plane, J. ACM 39 (1992), 1-54.

[CS89] K. Clarkson and P. Shor, Applications of random sampling in computational geometry II, Discrete Comput. Geom. 4 (1989), 387-421.

[KS96] J. Komlós and M. Simonovits, Szemerédi's regularity lemma and its applications in graph theory, in: Bolyai Society Mathematical Studies 2, em Combinatorics, Paul Erdős is Eighty (Volume 2), Budapest, 1996, 295-352.

[KM94] J. Kratochvíl and J. Matoušek, Intersection graphs of segments, Journal of Combinatorial Theory, Series B 62 (1994), 289-315.

[KST54] T. Kővári, V. T. Sós, and P. Turán, On a problem of K. Zarankiewicz, Colloq. Math. 3 (1954), 50-57. 
[L83] T. Leighton, Complexity Issues in VLSI, Foundations of Computing Series, MIT Press, Cambridge, MA, 1983.

[P91] J. Pach, Notes on geometric graph theory, in Discrete and Computational Geometry: Papers from the DIMACS Special Year (J. E. Goodman, R. Pollack, and W. Steiger, eds.) DIMACS Series in Discrete Mathematics and Theoretical Computer Science 6, American Mathematical Society, Providence, RI, 1991, 273-285.

[PA95] J. Pach and P.K. Agarwal, Combinatorial Geometry, J. Wiley \& Sons, New York, 1995.

[PS91] J. Pach and M. Sharir, On vertical visibility in arrangements of segments and the queue size in the Bentley-Ottmann line sweeping algorithm, SIAM J. Comput. 20 (1991), 460-470.

[PT97] J. Pach and G. Tóth, Graphs drawn with few crossings per edges, Combinatorica 17 (1997), 427-439.

[SH76] M. I. Shamos and D. Hoey, Geometric intersection problems, in: Proc. 17th Annual IEEE Symp. on Found. Comput. Sci. (1975), 151-162.

[S78] E. Szemerédi, Regular partitions of graphs, in: Problèmes combinatoires et théorie des graphes (Colloq. Internat. CNRS, Univ. Orsay, Orsay, 1976), Colloq. Internat. CNRS, 260, CNRS, Paris, 1978, 399-401. 